\title{
Populace Internet Participation in Food Safety Supervision
}

\author{
Qi Chunxia ${ }^{1, a}$, Meng Wenwen ${ }^{1, b}$ and Yao Xiaoyan ${ }^{1, c}$ \\ ${ }^{1}$ NO.201, Jufeng Lu, Shandong Foreign Trade Vocational College, Licang District, Qingdao, \\ Shandong, China \\ a45153304@qq.com, b181962188@qq.com, ${ }^{\text {c3 } 35366775 @ q q . c o m ~}$
}

Keywords: Food Safety Supervision, Populace Participation, Supervision System, Driving Force, Populace Internet Participation, the Internet Supervision

Abstract. Food safety supervision is a big problem in China, but the supervision system dominated by government is not enough for the vast market. The populace participation is very urgent because of various driving forces from internal needs and external needs. As the most effective platform for modern populace, the internet could bring five impacts including tools, channels, structure, management and effects. That means populace participate in the food safety supervision would be more effective and practical.

\section{Introduction}

Food safety is a very big problem in China. It relates to people's life and health as well as the social stability. Effective food supervision system is critical to ensure the food quality and safety. China has developed the supervision system dominated by the government which could supervise the food product market. However, the vast China market involve complex problems, China's food safety supervision system has shown the insufficient ability in the regulatory power.

As food safety directly to the populace, people have a positive willingness to participate in the supervision system. But the lack of sound mechanism and effective ways result to the poor participation effect. Meanwhile, more and more individuals are using the Internet to share information and communicate different problems. The Internet provide powerful and convenient tools for people, which means the populace can also use the Internet to share the food safety information and even participate in the supervision system.

Relevant academic documents[1-3] show that populace participation in the field of food safety supervision should be valued.

Some academic documents pay attention to the internet participation[4-7]. Scholars' research mainly concentrated on the internet influence for democratic politics and some surface strategies. There is still a lack of research on specific areas like food safety supervision. Researches for populace internet participation is at the initial stage,

\section{Driving Force of the Populace Participation in the Supervision System}

Driving Force from Populace themselves. The internal driving force of populace is analyzed by the data collected with Sojump-an online questionnaire research tool. Research found that populace hopes to participate in food safety supervision because of three related reasons. Firstly, people concerns about their own interest in consuming food. People want to know food security information include food ingredients, food quality and food safety certification. Secondly, there is a certain amount of democracy hope to learn more about food safety supervision. People want to show their attitude and express their suggestions in food safety supervision affaire. For example, when food safety problems or event happen, people want to give their evidence information and follow the supervision handling process. Many people like to concern about public's interests. Data also show that public satisfaction with the existing food safety supervision is not high, people wants to push the food safety supervision work to improve regulatory efficiency. 
Driving Force from government. China's food supply market is very huge and contains complex processes. But the regulatory power of the Chinese government is very limited. Food safety regulatory work often exists vacuum. The populace is the subject of food safety events. With their participation, government agencies can grasp safety issues at the first time and quickly respond to the food safety crisis so that they could reduce the damage level of the incident and establish good government figure. Otherwise, populace participation can promote the government departments to adjust the regulatory agencies or processes, to build a scientific and applicable regulatory organization system.

Driving Force from enterprises. From the angle of food supply chain, the relationship of the populaces and food production enterprises are supply and demand subjects. People have right to learn about the food safety information, and also they can report food safety issues timely to the enterprises. That's important for setting good corporate reputation and brand. At the same time, the public can also play the regulatory role of market to supervise poor-quality food enterprises and urge them to improve or withdraw.

Driving Force from testing and research organizations. When people come across food safety issues, specialized testing and research institutions can do help. They testing food ingredients and evaluate food quality safety for people. And people pay to support the operation of these institutions. Meanwhile, the needs of the social populace contain a variety of research topics, which help these organizations to collect research data and enrich the scientific research contents.

Driving Force from public medium. Social media has always played an very important role in kinds of incidents. They could spread the hazards knowledge of unsafe food, follow the food events process and call for effective solution for the food security crisis. They hope the populace participate in provide report materials timely. Furthermore, social medium can conduct public discussion and collect public opinions so that they can work more values for the society.

So, different subjects attributed to driving forces for populace's participate in the food safety supervision system. It shows the necessity of populace participate in the food safety supervision system.

The following figure shows the structure of the driving forces.

External forces

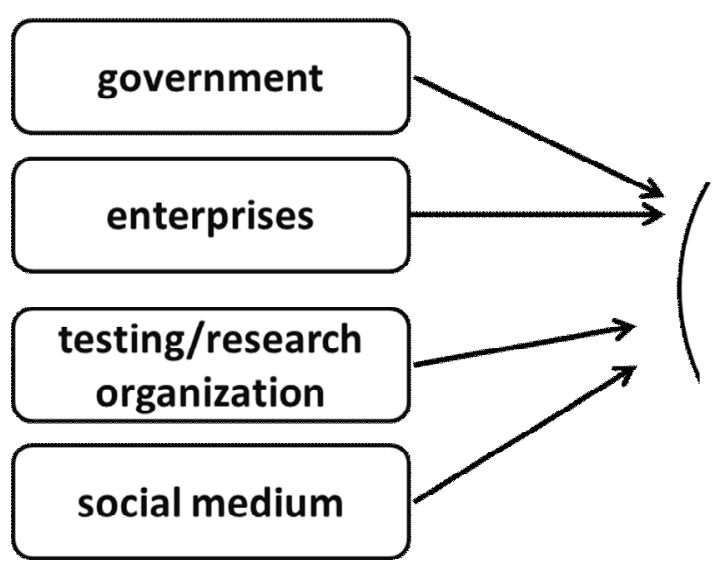

Fig. 1 the structure of driving forces for populace participation
Internal Forces

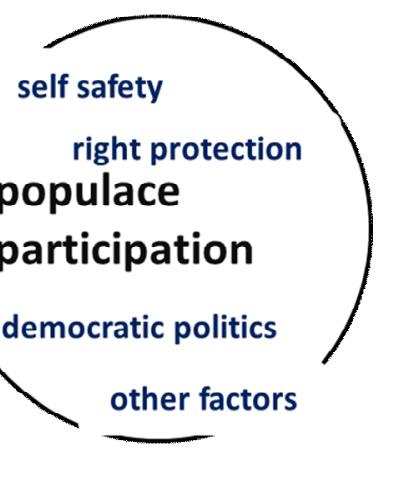

\section{The Internet powers for Populace Participation in the Supervision System}

Supervision system involves populace, government, enterprises, research organizations etc. Timely information communication is very critical. With outstanding technology highlights, the Internet has brought profound impacts for the populace participate in food safety supervision system. Considered the typical impact perspectives of the Internet, an Internet impact model for supervision system could be built. The following figure gives the model. 


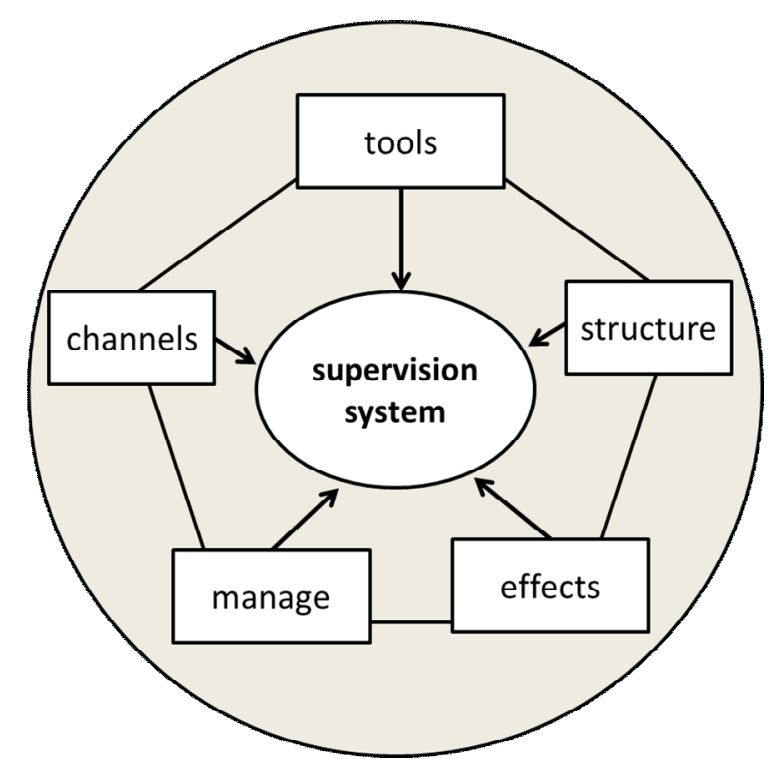

Fig. 2 the internet powers for supervision system

Tools. The Internet is a powerful technology system which includes World Wide Web technology, multimedia technology, server programming, data management technology etc. These technologies promote different developments of internet software which have shown their power in applications.

These technologies provide every possibility for populace participate in the food supervision system. Food safety related information could be completely delivery. Enterprises or research organizations could demonstrate food product information including appearance, components and quality with texts, images or videos. The Internet tools make populaces learn more about food safety information so that they could participate in supervision system more professional.

Channels. Besides tools, the Internet also provides people various communication channel that make communication easier. Web pages, email, BBS, Weibo, Weixin, social chatting software are the common applications in China.

Web pages with data collecting forms encourage populace to leave message if they have some food safety related questions. Email builds the bridge between populace and related organizations. Weibo and Weixin have got so popular for food safety information share because their quick spreading speed and high credibility friends circle. The mobile technology makes these channels more convenient for populace participate in the supervision system.

Structure. Compare with the traditional supervision system, the internet facility the direct information flow among related subjects so a kind of flat communication structure come into form.

Populace could interact directly with government, enterprises and organizations. Government, enterprises and organizations could reshape their business processes to more direct but not hierarchical. So, the communication structure of food supervision system could be changed with the internet.

Manage. The internet could trace the information flow and collect all kinds of data.

The IP tracing technology could tell the information source and decide the route of information flow. Once there's information about food safety event, the technology could quickly detect and tell to the supervision system. Database and data mining technology could store the food safety information and mining related incidents laws so the supervision system could run more efficiently.

Effects. The internet has great power in information spreading and audience gathering. It's the best communication environment for modern people.

It's easy for populace to search and browse food safety related information, to submit food safety questions and report food safety incidents. Virtual community and mobile social platform can record populace data and collect suggestions quickly. The effect would be suprising. 


\section{Significance of Populace Internet Participation in Food Safety Supervision}

Populace internet participation in food safety supervision is of great significance reflected in three levels. First level: it meets the internal demands and enthusiasm of populace as well as supplement the power of government, preventing regulatory loopholes and promote regulation performance. Second level: populace internet participation in food safety supervision could promote related enterprises, organizations and social media to improve quality and play values. Third level for populace internet participation lies in the democratic politics. Populace internet participation in food safety Internet will be conducive to the promotion of democratic participation in the network environment and the introduction of relevant policies and related policies to promote democratic politics. From these standpoints, populace internet participation would be sure to attribute more to food safety supervision.

However, there still need some efforts to promote the participation. Populace internet participation awareness needs to be strengthened. Related laws and regulations need to be studied and issued to encourage populace participation. Related platforms for the internet participation need to be developed. And China government should also consider how to combine the traditional supervision system and internet supervision system better.

\section{Conclusions}

Populace internet participation in food safety supervision is a new topic but very critical. It has enough driving forces and good internet support. It also integrates populace group power and internet technology advantages. The significance of the populace internet participation is very great. The country should positively study the internet participation laws and methods and issue them to promote the whole countries democratic politics.

\section{Acknowledgements}

This work was supported by Shandong Foreign Trade Vocational College.

\section{References}

[1] Xi Ming: public education-effective ways for food safety, agricultural product processing(2007.02)

[2] Zhou Zaohong: Game Analysis of Public Participation Management in the Food Safety in China, East China Economic Management,Vol.23(2009.09)

[3] Zhang Xuan: An empirical analysis on the problem of citizen participation in food safety supervision in Nanjing City, Prices Monthly.(2010.05)

[4] Oliver.Boyd.Barrett: Approaches to Media, Xinhua press(2004)

[5] Wang Yongsheng, Research on some problems of citizen's political participation in the network age, Dalian University of Technology (2000)

[6] Wu Qiong:Research on citizen participation in network conditions, China University of Geosciences(2009)

[7] Liang Yanxia:Research on food safety supervision from the perspective of network governance theory, Shantou University(2006) 